\title{
Fluid Resuscitation after Cardiac surgery: Is Hypertonic Saline or Hyperoncotic Albumin the Future?
}

\author{
Tiffany Otero ${ }^{1}$, Oscar Aljure ${ }^{2}$, and Soojie $\mathrm{Yu}^{1}$ \\ ${ }^{1}$ Banner University Medical Center Tuscon \\ ${ }^{2}$ University of Miami School of Medicine
}

June 22, 2020

\begin{abstract}
Background: Over-resuscitation in post-cardiac patients is associated with significant morbidity and mortality. Accordingly, there is a growing interest in concentrated human albumin and hypertonic saline for resuscitation in cardiac patients following revascularization surgery. In this article, we will review the use of hyperosmolar fluid therapies for resuscitation in post-cardiac surgical patients from the current literature. Methods: A literature search was conducted in MEDLINE (PubMed) utilizing keywords, narrowing publications to 2010-2019. Results: Patients receiving concentrated albumin after cardiac surgery required less fluid bolus therapy, less time on vasopressors and were found to have a less positive fluid balance compared to patients receiving crystalloids. The impact on cardiac output and mean arterial pressure in those given concentrated albumin compared to crystalloid boluses was inconsistent. There was no difference in mortality in those given $20 \%$ albumin compared to crystalloids post-revascularization. Hypertonic saline showed some positive immunomodulatory effects in patients post-revascularization. Studies identifying the impact of hypertonic saline on fluid balance and mortality compared to normal saline in patients following revascularization surgery are on-going. Conclusions: In this analysis, publications on resuscitation with hyperoncotic albumin and hypertonic saline in patients post-revascularization surgery were reviewed. While there is data in support of using these alternative fluid therapies in other critically ill patient populations, the limited literature in support of using concentrated albumin and hypertonic saline for resuscitation in following cardiac surgery is equivocal.
\end{abstract}

\section{Resuscitation with human albumin}

\section{Background}

The utility of providing volume expansion with colloids and their relative use in comparison to crystalloids has been studied extensively for decades [7, 25, 26]. First introduced in the 1940s, the first human protein isolated for clinical use was isooncotic $(4-5 \%)$ human albumin $[27,28]$. After this, other type of colloid solutions available for volume expansion became available in the 1970s, first with hydroxyethyl starches (HES), followed by other types of synthetic colloids [26, 29].

Since their inception, volume expansion with synthetic colloids have been studied extensively in the ICU through a number of well-designed randomized controlled trials, systematic reviews and meta-analyses [3033]. These studies demonstrate that resuscitation in the ICU with artificial colloids is associated with poorer patient outcomes and increased costs when compared with crystalloids [30, 31, 34-37]. In contrast, the literature investigating resuscitation with natural colloids (i.e. human albumin) suggests fluid therapy with human albumin may be superior to crystalloids in certain patient populations [19, 20, 38-41].

\section{Isooncotic human albumin}

The theoretical value of fluid therapy with isooncotic human albumin over crystalloids is multifactorial [20, $27,42]$. Physiologically, albumin is the primary determinant of intravascular osmotic pressure, aids in the 
transport of several compounds, serves as a native free radical scavenger and possess both anti-inflammatory and antioxidant properties [27, 42-44].

As a volume expander, isooncotic human albumin is theorized to provide intravascular volume expansion more efficiently than crystalloids [24]. Colloids are hypothesized to remain in the intravascular space for a more sustained period of time [25, 45], and provide volume expansion without a significant chloride burden when compared to both isotonic saline and balanced crystalloid solutions [20,27]. The latter is particularly relevant given the growing body of evidence that indicates elevated chloride levels are harmful in the critically ill [46-48].

The potential risks of resuscitation in the ICU with human albumin has also been investigated [49-51]. When compared to crystalloids, there is evidence to suggest resuscitation with human albumin may be related to coagulation abnormalities [50], increased need for blood transfusions [50, 52], anaphylactoid reactions [40], and acute kidney injury $[51,53]$. Moreover, there has been no consistent evidence that suggests resuscitation with albumin improves mortality, number of ventilator free days or length of hospital stay [52]. In addition to these potential clinical sequelae, human albumin costs significantly more in comparison to saline and balanced intravenous fluids [12, 49, 52].

Resuscitation with hyperoncotic human albumin following cardiovascular surgery

The advantages and risks of resuscitation with isooncotic albumin are potentially of even greater significance when volume expansion is achieved with hyperosmolar albumin [45, 54]. Prospective studies performed in various ICU settings have indicated that the volume-expanding effect of hyperosmolar albumin is twice that of $5 \%$ human albumin $[45,55]$ with results demonstrating a less positive fluid balance in patients resuscitated with hyperoncotic albumin $[43,45,55]$.

Similar investigations in patients following cardiac surgery had not been performed until recently. In a prospective, sequential, open-label study by Wigmore, et al., authors aimed to compare the effects of FBT using $20 \%$ albumin $(200 \mathrm{~mL}$ bolus) versus crystalloid $(500 \mathrm{~mL}$ bolus) on fluid balance, hemodynamic markers and clinical course in the ICU in a cohort of post-cardiac patients $(n=100)$ [20]. FBT was given in response to clinical signs of hemodynamic instability or in response to a low cardiac index $\left(<2.2 \mathrm{~L} / \mathrm{min} / \mathrm{m}^{2}\right)[20]$. Patients in the albumin group were noted to have a less positive fluid balance in the first 24 hours $(\mathrm{p}=0.001)$, and required fewer FBT compared to those in the crystalloid group $(\mathrm{p}<0.001)$ [20]. Further, individuals in the concentrated albumin group required vasopressors for a shorter period of time and had a shorter length of ICU stay compared to their study counterparts $(\mathrm{p}=0.048)$ [20]. Despite these findings, there was no appreciable difference between the two groups in cardiac output (CO), mean arterial pressure (MAP) or central venous pressure (CVP) following the first fluid bolus [20]. Furthermore, this study showed no difference in mortality between study groups [20].

In a smaller prospective observational study by Cutuli, et al., authors investigated the effects of $20 \%$ albumin boluses on cardiac index (CI) and MAP in a cohort of twenty post-cardiac patients [24]. Patients included in this single center analysis were those admitted to the ICU following an on-pump cardiovascular surgery, who were given a bolus of $20 \%$ albumin within the first twelve hours of ICU admission [24]. The clinical indications of when to administer FBT was similar to the study conducted by Wigmore, et al [20, 24]. After the administration of FBT, $55 \%$ of the study cohort demonstrated an appreciable increase in MAP ( $>10 \%)$ [24]. This effect dissipated in nearly half of the responders within 30 minutes of their FBT, returning to their MAP baseline [24]. Interestingly, the impact FBT had on CI for the study cohort varied from these results [24]. While only $25 \%$ of participants had a significant increase in CI $(>15 \%)$ immediately after receiving hyperoncotic albumin, $25 \%$ of the cohort demonstrated a delayed increase in CO, one that persisted beyond 30 minutes [24]. One explanation to these incongruent findings is the notion that the effect of hyperoncotic albumin peaks at 30 minutes after administration [24].

The parameters used when FBTs were given in these two analyses highlights the individualized practice of fluid resuscitation in the perioperative setting [12]. In a retrospective, non-interventional descriptive study performed at a tertiary care medical center, Torbic, et al. aimed to describe the prescribing practice of 
concentrated albumin within the ICUs at their institution $(n=2,066)$ [12]. During the study period, the majority of the hyperoncotic albumin administered within their institution occurred in the cardiovascular ICU (CVICU) in patients following cardiac surgery $(\mathrm{n}=1509,73 \%)$ [12]. In this analysis, authors describe the medical conditions that may benefit from the administration of human albumin, where the use of hyperosmotic albumin is potentially superior to isooncotic albumin [12]. The evidence-based indications include post-subarachnoid hemorrhage vasospasm, large-volume paracentesis, spontaneous bacterial peritonitis and hepatorenal syndrome $[12,56-58]$. Based on these indications, authors discussed the hyperosmolar albumin administration practices in the CVICU, emphasizing the use of hypertonic albumin in response to hypotension, low urine output or perceived hypovolemia to be inappropriate and unsupported by the current literature [12]. From a health care utilization standpoint, the authors also discuss the enormous cost associated with the use of concentrated human albumin [12].

In an effort to identify best practice and further delineate the appropriateness of the routine administration of concentrated albumin, Rabin, et al., studied the effect of restrictive albumin practices following cardiac surgery over a thirteen month period [52]. During this retrospective analysis, authors investigated the use of hyperoncotic albumin in patients admitted to the CVICU following cardiac surgery before and after institutional guidelines limiting the use of albumin administration $(n=1,401)$ [52]. Outcomes of interest included the amount of albumin use, number of blood transfusions, ventilator free days, mortality and length of hospital stay [52]. During the first nine months of the study period, there were no restrictions in albumin administration [52]. Following the implementation of the restrictive guidelines, the use of albumin was limited to patients that required more than three liters of crystalloids 24 hours after surgery [52]. Following the initiation of restrictive guidelines, authors found the amount of hyperoncotic albumin given decreased from a mean of 280 monthly doses to a mean of 101 monthly doses $(\mathrm{p}<0.001)$ [52]. This decrease in 180 albumin doses equated to more than $\$ 45,000$ saved per month [52]. Authors found no difference in average length of stay, mortality, ventilator free days or number of transfusions required between the two study period [52].

\section{Resuscitation with Crystalloid}

\section{Background}

Crystalloid fluids have been used as a method of resuscitation since the cholera epidemic in the 1830's [59]. Crystalloids are a family of aqueous solutions containing different concentrations of ions and small organic molecules [60]. These solutions are classified as physiologically unbalanced/nonbuffered (e.g. normal saline), physiologically balanced/buffered (e.g. lactate, acetate, maleate) or classified by their tonicity relative to human plasma (isotonic, hypotonic or hypertonic) [59, 61, 62].

\section{Unbalanced Crystalloid Solutions}

Unbalanced/nonbuffered solution mainly refer to $0.9 \%$ saline or normal saline (NS) [59]. The composition of normal saline contains supraphysiological concentrations of chloride, which often results in a hyperchloremic state in those resuscitated with NS [59]. As mentioned above, the impact of hyperchloremia in the critically ill is of great clinical significance [46-48]. Specifically, hyperchloremia has been found to be associated with acute kidney injury [47], non-anion gap metabolic acidosis [63], decreased ventilator free days [64], bleeding and mortality in the perioperative period [59]. Despite these clinical sequelae, NS is used frequently following CABG procedures for perioperative resuscitation, due to its wide availability and relative low cost [15].

\section{Balanced Crystalloid solutions}

Balanced crystalloids are comprised of solutes that more closely resemble human plasma [59, 60]. Based on the composition of the crystalloid, the solutes will contribute to intravascular volume expansion, all while counteracting fluid shifts into the extracellular space by providing oncotic pressure[59]. While these solutions are more "physiologic" when compared to NS, balanced solutions are not perfect [59]. The benefit of resuscitation with buffered solutions compared to nonbuffered crystalloid solutions in noncardiac surgery patients remains equivocal [63, 65-69]. In a double-blinded, randomized, double-crossover and prospective 
cohort study in post cardiac surgery patients, Reddy et al. studied post-operative bleeding in patients resuscitated with Plasma-lyte (Baxter International Inc., Deerfield, IL) compared to NS ( $\mathrm{n}=475$ in Plasmalyte group and $n=479$ in normal saline group) [61]. Through this analysis, authors found the transfusion rate was significantly higher in the first three days in the ICU in those receiving Plasma-Lyte compared to the control NS group $(\mathrm{p}=0.008)$ [61]. Moreover, investigators found individuals in the Plasma-Lyte group had significantly higher INR's

$(\mathrm{p}=0.04)$ and an overall longer hospital length of stay compared to those resuscitated with NS ( $\mathrm{p}=0.04)$ [61].

The pathophysiology of these clinical effects in buffered solutions is not well understood in-vivo [61, 70]. When further analyzing other balanced solutions, there is scarce evidence that suggests resuscitation with intravenous fluids buffered with acetate or lactate impacts inotrope usage, cumulative amount of fluids necessary, or acid base profiles [17].

The use of crystalloids following cardiac surgery

In a multicenter observational study by Park, et al., researchers showed that cardiac patients often receive 4-5L of fluid in the first 24 hours in the ICU post-operatively [71], with crystalloids being the most commonly used solution $[13,71]$. Crystalloids are utilized due to its low-cost, ease of use, and multiple studies showing no significant evidence of improved outcomes when administered albumin over crystalloid[38, 49, 52, 72]. In comparison to colloids, multiple studies indicate that more crystalloid fluid administration is needed to create similar changes in hemodynamic goals $[49,52,62]$.

For post-operative cardiac patients, increase fluid administration can be associated with acute renal dysfunction, increased length of stay in the ICU, and mortality [16, 73]. Stein, et al. found a moderate association between length of ICU stay and fluid overload $(\mathrm{r}=0.57, P<0.001)[16]$. They observed that $10 \%$ fluid overload had a significant and independent effect on combined events including death, infection, arrhythmia, bleeding and pulmonary edema (OR 4.43; CI 2.08 to 9.14; $P<0.001)[16]$.

Resuscitation with hypertonic saline following cardiac surgery

To modulate the risks of volume overload with isotonic crystalloid solutions, resuscitation with hypertonic saline (HS) is of increased clinical interest in the perioperative setting [21, 74]. HS has been used for over 30 years in the treatment of hypovolemic and septic shock, burn injuries, hyponatremia and increased intercranial pressure [74-76]. Due to a higher tonicity relative to human plasma, HS creates an osmotic gradient that promotes fluid to shift from the extracellular space to the intravascular space [74]. This relative increase in intravascular volume serves to increase preload [60,74, 76, 77]. Thus, when compared to equivalent volumes of NS, resuscitation with HS results in a more profound increase in MAP, CO and stroke volume in those who are critically ill [74, 78].

Beyond its hemodynamic effects, resuscitation with HS may also have an impact on systemic inflammation [76]. HS has been found to blunt neutrophil activity, cytokine production, and pro-inflammatory markers (e.g. IL-G and TNF-alpha) all while upregulating the activity of anti-inflammatory markers, such as IL-10 [76]. This is of particular significance in patients undergoing cardiac surgery, given the degree of systemic inflammation and complement activation that occurs during extracorporeal circulation [76].

To investigate this further, Yousefshahi et al. conducted a randomized, double-blinded clinical trial comparing inflammatory markers predictive of AKI in individuals given HS compared to NS prior to their CABG procedure $(\mathrm{n}=40)$. Inflammatory markers of interest (neutrophil gelatinase-associated lipocalin (NGAL) and cystatin C) were measured before cardiac surgery, 24 hours and 48 hours post-CABG [79]. While investigators did not find a statistically significant decrease in NGAL or cystatin C in those given HS compared to NS ( $\mathrm{p}=0.792$ and $\mathrm{p}=0.874$, respectively) [79], other analyses have demonstrated increased urinary output independent of diuretic usage and less weight gain post-CABG in those given HS compared to NS [74, 80].

To further investigate HS as a resuscitation fluid in patients following elective cardiac surgery, Pfortmueller et al. recently published a study protocol for a single-center, prospective randomized controlled clinical 
trial comparing post-operative FBT with HS to NS in patients post-CABG[21] The primary objective of The Hypertonic Saline for Fluid resuscitation after cardiac surgery (HERACLES) trial is to determine if resuscitation with HS results in less total fluid volume administered when compared to NS [21] The HERCULES trial further aims to investigate whether the use of HS results in a difference in post-operative weight gain, cumulative vasopressor use, or urinary output when compared to NS after cardiac surgery [21]. Exploratory outcomes described in the HERCULES protocol also includes comparing

ICU and hospital mortality, duration of ventilator dependence, and need for renal replacement therapy between study groups [21]. While results from the HERCULES trial are pending, this robust study highlights the paucity of literature currently available describing the use of HS for resuscitation in those following revascularization procedures [21].

\section{Discussion}

In this review, low volume resuscitation with hyperoncotic human albumin and hypertonic saline in patients following revascularization cardiac surgery was explored. In the literature currently available, the use of hyperoncotic albumin for post-CABG resuscitation has been published on more extensively when compared to HS, with the HERCULES trial being the major randomized controlled trial in progress investigating the role of HS in patients post-CABG [21]. The objectives of both bodies of literature aim to delineate whether or not resuscitation with concentrated albumin and saline improved hemodynamics more efficiently than their isooncotic fluid counterparts [20, 21, 24]. In addition, these analyses further attempted to identify whether resuscitation with these alternative, concentrated intravenous fluids impacted overall fluid balance $[20,21]$.

In total, the results from the available data did not consistently show hyperoncotic colloid to be a more superior volume expander when compared to crystalloid solutions in patients following cardiac revascularization procedure $[20,24]$. Moreover, the current evidence did not demonstrate an appreciable difference in mortality in post-CABG patients resuscitated with 20-25\% human albumin when compared to crystalloid fluid therapy [20,24]. Similarly, while resuscitation with HS in the critically ill has been shown to increase $\mathrm{CO}$ and MAP to a greater extent compared to a similar volume of NS [74], this has yet to be determined in patients following CABG-procedure [20, 21].

While the current evidence in support of using concentrated human albumin and saline remains equivocal, the literature available for interpretation has its limitations. First, the number of publications studying the use of HS and concentrated albumin in post-CABG patients is sparse, composed of small patient cohorts. Moreover, the available studies included in this analysis used static hemodynamic parameters (i.e CO, CI, CVP, Urine output and MAP) to quantify hemodynamic changes, metrics known to be poor predictors of fluid responsiveness $[60,81]$. Additionally, these studies did not comment on the type of fluid patients were given pre-operatively, intra-operatively, or the type of fluid used to prime the cardiopulmonary bypass circuit, which could have impacted their overall results. Finally, while the 2019 Enhanced Recovery after Cardiac Surgery guidelines recommends goal directed fluid therapy (an algorithmic approach to resuscitation), no universal protocol exists for post-operative resuscitation following CABG-procedure [82]. Accordingly, the studies included in this review had unique protocols to guide the administration of intravenous therapy.

In lieu of the static hemodynamic markers used in the available studies, future analyses could instead use functional dynamic measures of stroke volume, such as pulse pressure variation, stroke volume variation, and systolic pressure variation to more reliably quantify fluid responsiveness [60, 81, 83]. In addition to this, echocardiography could be used to guide clinical practice by evaluating biventricular function and volume assessment, an imaging modality that would more reliably quantify fluid responsiveness [84]. Finally, the passive leg raise (PLR) could be of use to aid clinicians in determining the need for FBT in future studies on HS and hyperoncotic albumin in post-CABG patients [81, 85]. Future studies utilizing more accurate measurements of volume status, fluid responsiveness and cardiac output could therefore further determine the role of concentrated human albumin and saline in patients following revascularization cardiac surgery, through prospective randomized controlled trials. 


\section{References}

1. Jovin, D.G., G.J. Katlaps, and K.F. Sumption, Coronary artery bypass graft markers: history, usage, and effects. Gen Thorac Cardiovasc Surg, 2020. 68 (5): p. 453-458.

2. Head, S.J., et al., Mortality after coronary artery bypass grafting versus percutaneous coronary intervention with stenting for coronary artery disease: a pooled analysis of individual patient data.Lancet, 2018. 391 (10124): p. 939-948.

3. Habib, R.H., et al., CABG Versus PCI: Greater Benefit in Long-Term Outcomes With Multiple Arterial Bypass Grafting. J Am Coll Cardiol, 2015. 66 (13): p. 1417-27.

4. Li, C., et al., Early negative fluid balance is associated with lower mortality after cardiovascular surgery. Perfusion, 2018.33 (8): p. 630-637.

5. Ghotkar, S.V., et al., Preoperative calculation of risk for prolonged intensive care unit stay following coronary artery bypass grafting. J Cardiothorac Surg, 2006. 1 : p. 14.

6. Aronson, S., P. Nisbet, and M. Bunke, Fluid resuscitation practices in cardiac surgery patients in the USA: a survey of health care providers. Perioper Med (Lond), 2017. 6 : p. 15.

7. Martin, C., et al., Choice of fluids in critically ill patients. BMC Anesthesiol, 2018. 18 (1): p. 200.

8. Ogbu, O.C., D.J. Murphy, and G.S. Martin, How to avoid fluid overload. Curr Opin Crit Care, 2015. 21 (4): p. $315-21$.

9. Pradeep, A., et al., High volumes of intravenous fluid during cardiac surgery are associated with increased mortality. HSR Proc Intensive Care Cardiovasc Anesth, 2010. 2 (4): p. 287-96.

10. Ricci, Z. and S. Romagnoli, The issue of fluid balance and mortality. HSR Proc Intensive Care Cardiovasc Anesth, 2010.2 (4): p. 245-7.

11. Levin, M.A., et al., Early on-cardiopulmonary bypass hypotension and other factors associated with vasoplegic syndrome.Circulation, 2009. 120 (17): p. 1664-71.

12. Torbic, H., et al., Evaluation of Albumin 25\% Use in Critically Ill Patients at a Tertiary Care Medical Center. Hospital Pharmacy, 2020. 55 (2): p. 90-95.

13. Menger, J., et al., Postoperative management of patients undergoing cardiac surgery in Austria : A national survey on current clinical practice in hemodynamic monitoring and postoperative management. Wien Klin Wochenschr, 2018. 130 (23-24): p. 716-721.

14. Marik, P.E., X. Monnet, and J.L. Teboul, Hemodynamic parameters to guide fluid therapy. Ann Intensive Care, 2011.1 (1): p. 1.

15. Parke, R.L., et al., Intravenous fluid use after cardiac surgery: a multicentre, prospective, observational study. Crit Care Resusc, 2014. 16 (3): p. 164-9.

16. Stein, A., et al., Fluid overload and changes in serum creatinine after cardiac surgery: predictors of mortality and longer intensive care stay. A prospective cohort study. Crit Care, 2012.16 (3): p. R99.

17. Pfortmueller, C.A., et al., Fluid management in patients undergoing cardiac surgery: effects of an acetate- versus lactate-buffered balanced infusion solution on hemodynamic stability (HEMACETAT). Crit Care, 2019. 23 (1): p. 159.

18. Lee, E.H., et al., Effect of Exogenous Albumin on the Incidence of Postoperative Acute Kidney Injury in Patients Undergoing Off-pump Coronary Artery Bypass Surgery with a Preoperative Albumin Level of Less Than $4.0 \mathrm{~g} / \mathrm{dl}$. Anesthesiology, 2016. 124 (5): p. 1001-11.

19. Sedrakyan, A., et al., Volume expansion with albumin decreases mortality after coronary artery bypass graft surgery. Chest, 2003.123 (6): p. 1853-7. 
20. Wigmore, G.J., et al., 20\% Human Albumin Solution Fluid Bolus Administration Therapy in Patients After Cardiac Surgery (the HAS FLAIR Study). J Cardiothorac Vasc Anesth, 2019. 33 (11): p. 2920-2927.

21. Pfortmueller, C.A., et al., Hypertonic saline for fluid resuscitation after cardiac surgery (HERACLES): study protocol for a preliminary randomised controlled clinical trial. Trials, 2019.20 (1): p. 357.

22. Bueno, R., et al., Effects of hypertonic saline-dextran solution in cardiac valve surgery with cardiopulmonary bypass. Ann Thorac Surg, 2004. 77 (2): p. 604-11; discussion 611.

23. Järvelä, K. and S. Kaukinen, Hypertonic saline (7.5\%) after coronary artery bypass grafting. Eur J Anaesthesiol, 2001.18 (2): p. 100-7.

24. Cutuli, S.L., et al., Haemodynamic effect of a $20 \%$ albumin fluid bolus in post-cardiac surgery patients. Crit Care Resusc, 2020.22 (1): p. 15-25.

25. Martin, G.S. and P. Bassett, Crystalloids vs. colloids for fluid resuscitation in the Intensive Care Unit: A systematic review and meta-analysis. J Crit Care, 2019. 50 : p. 144-154.

26. Haupt, M.T. and E.C. Rackow, Colloid osmotic pressure and fluid resuscitation with hetastarch, albumin, and saline solutions.Crit Care Med, 1982. 10 (3): p. 159-62.

27. Vincent, J.L., et al., Albumin administration in the acutely ill: what is new and where next? Crit Care, 2014. 18 (4): p. 231.

28. Rozga, J., T. Piatek, and P. Malkowski, Human albumin: old, new, and emerging applications. Ann Transplant, 2013. 18 : p. 205-17.

29. Salmon, J.B. and M.G. Mythen, Pharmacology and physiology of colloids. Blood Rev, 1993. 7 (2): p. 114-20.

30. Mutter, T.C., C.A. Ruth, and A.B. Dart, Hydroxyethyl starch (HES) versus other fluid therapies: effects on kidney function.Cochrane Database Syst Rev, 2013(7): p. Cd007594.

31. Taylor, C., et al., Hydroxyethyl starch versus saline for resuscitation of patients in intensive care: longterm outcomes and cost-effectiveness analysis of a cohort from CHEST. Lancet Respir Med, 2016. 4 (10): p. 818-825.

32. Li, B., et al., Resuscitation Fluids in Septic Shock: A Network Meta-Analysis of Randomized Controlled Trials. Shock, 2019.

33. Haase, N., et al., Hydroxyethyl starch 130/0.38-0.45 versus crystalloid or albumin in patients with sepsis: systematic review with meta-analysis and trial sequential analysis. Bmj, 2013. 346 : p. f839.

34. Bayer, O., et al., Effects of fluid resuscitation with synthetic colloids or crystalloids alone on shock reversal, fluid balance, and patient outcomes in patients with severe sepsis: a prospective sequential analysis. Crit Care Med, 2012. 40 (9): p. 2543-51.

35. Zarychanski, R., et al., Association of hydroxyethyl starch administration with mortality and acute kidney injury in critically ill patients requiring volume resuscitation: a systematic review and meta-analysis. Jama, 2013. 309 (7): p. 678-88.

36. Hartog, C.S., M. Bauer, and K. Reinhart, The efficacy and safety of colloid resuscitation in the critically ill. Anesth Analg, 2011. 112 (1): p. 156-64.

37. Perner, A., et al., Hydroxyethyl starch 130/0.42 versus Ringer's acetate in severe sepsis. N Engl J Med, 2012. 367 (2): p. 124-34.

38. Finfer, S., et al., A comparison of albumin and saline for fluid resuscitation in the intensive care unit. $\mathrm{N}$ Engl J Med, 2004.350 (22): p. 2247-56. 
39. Delaney, A.P., et al., The role of albumin as a resuscitation fluid for patients with sepsis: a systematic review and meta-analysis.Crit Care Med, 2011. 39 (2): p. 386-91.

40. Kingeter, A.J., et al., Association between albumin administration and survival in cardiac surgery: a retrospective cohort study. Can J Anaesth, 2018. 65 (11): p. 1218-1227.

41. Xu, J.Y., et al., Comparison of the effects of albumin and crystalloid on mortality in adult patients with severe sepsis and septic shock: a meta-analysis of randomized clinical trials. Crit Care, 2014.18 (6): p. 702.

42. Ferrer, R., et al., Non-oncotic properties of albumin. A multidisciplinary vision about the implications for critically ill patients. Expert Rev Clin Pharmacol, 2018. 11 (2): p. 125-137.

43. Caironi, P., et al., Albumin replacement in patients with severe sepsis or septic shock. N Engl J Med, 2014. 370 (15): p. 1412-21.

44. Quinlan, G.J., G.S. Martin, and T.W. Evans, Albumin: biochemical properties and therapeutic potential. Hepatology, 2005.41 (6): p. 1211-9.

45. Martensson, J., et al., Small volume resuscitation with 20\% albumin in intensive care: physiological effects : The SWIPE randomised clinical trial. Intensive Care Med, 2018. 44 (11): p. 1797-1806.

46. Shaw, A.D., et al., Major complications, mortality, and resource utilization after open abdominal surgery: 0.9\% saline compared to Plasma-Lyte. Ann Surg, 2012.255 (5): p. 821-9.

47. Yunos, N.M., et al., Chloride-liberal vs. chloride-restrictive intravenous fluid administration and acute kidney injury: an extended analysis. Intensive Care Med, 2015. 41 (2): p. 257-64.

48. Yunos, N.M., et al., Association between a chloride-liberal vs chloride-restrictive intravenous fluid administration strategy and kidney injury in critically ill adults. Jama, 2012. 308 (15): p. 1566-72.

49. Fink, R.J., et al., Cohort Study of Albumin versus Lactated Ringer's for Postoperative Cardiac Surgery Fluid Resuscitation in the Intensive Care Unit. Pharmacotherapy, 2018. 38 (12): p. 1241-1249.

50. Skhirtladze, K., et al., Comparison of the effects of albumin 5\%, hydroxyethyl starch 130/0.4 6\%, and Ringer's lactate on blood loss and coagulation after cardiac surgery. Br J Anaesth, 2014.112 (2): p. 255-64.

51. Schortgen, F., et al., The risk associated with hyperoncotic colloids in patients with shock. Intensive Care Med, 2008.34 (12): p. 2157-68.

52. Rabin, J., et al., Restricted Albumin Utilization Is Safe and Cost Effective in a Cardiac Surgery Intensive Care Unit. Ann Thorac Surg, 2017. 104 (1): p. 42-48.

53. Frenette, A.J., et al., Albumin administration is associated with acute kidney injury in cardiac surgery: a propensity score analysis. Crit Care, 2014. 18 (6): p. 602.

54. Jacob, M., et al., The intravascular volume effect of Ringer's lactate is below 20\%: a prospective study in humans. Crit Care, 2012.16 (3): p. R86.

55. Margarson, M.P. and N.C. Soni, Changes in serum albumin concentration and volume expanding effects following a bolus of albumin 20\% in septic patients. Br J Anaesth, 2004.92 (6): p. 821-6.

56. Runyon, B.A., Introduction to the revised American Association for the Study of Liver Diseases Practice Guideline management of adult patients with ascites due to cirrhosis 2012. Hepatology, 2013.57 (4): p. 1651-3.

57. Connolly, E.S., Jr., et al., Guidelines for the management of aneurysmal subarachnoid hemorrhage: a guideline for healthcare professionals from the American Heart Association/american Stroke Association. Stroke, 2012. 43 (6): p. 1711-37.

58. Evidence-based colloid use in the critically ill: American Thoracic Society Consensus Statement. Am J Respir Crit Care Med, 2004.170 (11): p. 1247-59. 
59. Reddy, S., et al., Choice of Fluid Therapy and Bleeding Risk After Cardiac Surgery. J Cardiothorac Vasc Anesth, 2016.30 (4): p. 1094-103.

60. Shaw, A. and K. Raghunathan, Fluid management in cardiac surgery: colloid or crystalloid? Anesthesiol Clin, 2013.31 (2): p. 269-80.

61. Reddy, S.K., et al., Effect of 0.9\% Saline or Plasma-Lyte 148 as Crystalloid Fluid Therapy in the Intensive Care Unit on Blood Product Use and Postoperative Bleeding After Cardiac Surgery. J Cardiothorac Vasc Anesth, 2017. 31 (5): p. 1630-1638.

62. Annane, D., et al., Effects of fluid resuscitation with colloids vs crystalloids on mortality in critically ill patients presenting with hypovolemic shock: the CRISTAL randomized trial. JAMA, 2013. 310 (17): p. 1809-17.

63. Reddy, S.K., et al., A protocol for the $0.9 \%$ saline versus Plasma-Lyte 148 for intensive care fluid therapy (SPLIT) study. Crit Care Resusc, 2014. 16 (4): p. 274-9.

64. Krajewski, M.L., et al., Meta-analysis of high-versus low-chloride content in perioperative and critical care fluid resuscitation. Br J Surg, 2015. 102 (1): p. 24-36.

65. Kim, S.Y., et al., Comparison of the effects of normal saline versus Plasmalyte on acid-base balance during living donor kidney transplantation using the Stewart and base excess methods. Transplant Proc, 2013. 45 (6): p. 2191-6.

66. Orbegozo Cortes, D., A. Rayo Bonor, and J.L. Vincent, Isotonic crystalloid solutions: a structured review of the literature. Br J Anaesth, 2014. 112 (6): p. 968-81.

67. Young, J.B., et al., Saline versus Plasma-Lyte A in initial resuscitation of trauma patients: a randomized trial. Ann Surg, 2014.259 (2): p. 255-62.

68. Reddy, S.K., et al., Overview of the study protocols and statistical analysis plan for the Saline versus Plasma-Lyte 148 for Intravenous Fluid Therapy (SPLIT) research program. Crit Care Resusc, 2015. 17 (1): p. 29-36.

69. Young, P., et al., Effect of a Buffered Crystalloid Solution vs Saline on Acute Kidney Injury Among Patients in the Intensive Care Unit: The SPLIT Randomized Clinical Trial. JAMA, 2015.314 (16): p. 170110.

70. Davies, P.G., et al., Plasma acetate, gluconate and interleukin-6 profiles during and after cardiopulmonary bypass: a comparison of Plasma-Lyte 148 with a bicarbonate-balanced solution.Crit Care, 2011. 15 (1): p. R21.

71. Parke, R.M., S. P.; Gilder, E; McCarthy, L. W., Intravenous Fluid use after cardiac surgery: a multicentre, prospective, observational study. Critical Care and Resuscitation, 2014.16 (3): p. 164-169.

72. Lewis, S.R., et al., Colloids versus crystalloids for fluid resuscitation in critically ill people. Cochrane Database Syst Rev, 2018. 8 : p. CD000567.

73. Verheij, J., et al., Effect of fluid loading with saline or colloids on pulmonary permeability, oedema and lung injury score after cardiac and major vascular surgery. Br J Anaesth, 2006.96 (1): p. 21-30.

74. Pfortmueller, C.A. and J.C. Schefold, Hypertonic saline in critical illness - A systematic review. J Crit Care, 2017. 42 : p. 168-177.

75. Strandvik, G.F., Hypertonic saline in critical care: a review of the literature and guidelines for use in hypotensive states and raised intracranial pressure. Anaesthesia, 2009. 64 (9): p. 990-1003.

76. Mazandarani, M., et al., Comparison of hypertonic saline versus normal saline on cytokine profile during CABG. Daru, 2012.20 (1): p. 49. 
77. Mustafa, I. and X.M. Leverve, Metabolic and hemodynamic effects of hypertonic solutions: sodium-lactate versus sodium chloride infusion in postoperative patients. Shock, 2002. 18 (4): p. 306-10.

78. de Mola, R.F., J.; Pescioto, V. R.; Lovatti, D. H.; Dallan, L.A.O.D; Rocha-e-Silva, R.; , Safety and efficacy of hypertonic saline versus isotonic saline solution in off-pump coronary artery bypass grafting. MedicalExpress, 2014. 1 (1): p. 27-30.

79. Yousefshahi, F., et al., Effect of Hypertonic Saline Infusion versus Normal Saline on Serum NGAL and Cystatin C Levels in Patients Undergoing Coronary Artery Bypass Graft. J Tehran Heart Cent, 2013.8 (1): p. 21-7.

80. Paterna, S., et al., Short-term effects of hypertonic saline solution in acute heart failure and long-term effects of a moderate sodium restriction in patients with compensated heart failure with New York Heart Association class III (Class C) (SMAC-HF Study). Am J Med Sci, 2011. 342 (1): p. 27-37.

81. Cherpanath, T.G., et al., Basic concepts of fluid responsiveness. Neth Heart J, 2013. 21 (12): p. 530-6.

82. Gregory, A.J., et al., Enhanced Recovery After Cardiac Surgery (ERAS Cardiac) Recommendations: An Important First Step-But There Is Much Work to Be Done. J Cardiothorac Vasc Anesth, 2020. 34 (1): p. $39-47$.

83. Lansdorp, B., et al., Dynamic indices do not predict volume responsiveness in routine clinical practice. Br J Anaesth, 2012.108 (3): p. 395-401.

84. Lazzeri, C. and A. Peris, Fluid responsiveness raises many questions-echocardiography may be the answer. J Thorac Dis, 2017.9 (4): p. 946-949.

85. Monnet, X., P.E. Marik, and J.L. Teboul, Prediction of fluid responsiveness: an update. Ann Intensive Care, 2016. 6 (1): p. 111. 